Le traitement à la soude a été effectué quotidiennement à la brosse et pendant quelques minutes à chaque action. Cependant, les échantillons préparés en usine par les fournisseurs ont été trempés dans les bains de soude et non brossés.

Le traitement à l'acide azotique a eu lieu par aspersion.

Sur les échantillons de grandes dimensions, on n'a procédé au lavage que sur une moitié de chaque surface considérée, eeci permettant la comparaison des parties traitées et non traitées.

Nos constatations nous conduisent aux avis ci-dessous qui n'engagent évidemment que-les catégories de peintures étudiées dans le cadre des marques choisies.

Les peintures aux résines vinyliques et les peintures au caoutchouc chloré donnent satisfaction, mais il semble que les dernières soient d'une application moins facile. Les peintures phénoliques résistent moins aux acides et bases diluśes que les précédentes, leur applieation est très aisée. Dans la mesure où la détrempe n'existe pas pour les peintures phénoliques, on peut les utiliser pour des revêtements de murs, mais l'utilisation dans deś baes de stockage de produits acides ou d'alcali ne nous parait pas à conseiller.

Dans tous les cas, une couche de protection antirouille est nécessaire sur tôle.

Sur bois, nos essais sont assez réduits, les résultats pouvant varier avec l'essence choisie. On attachera pour ce matériau une grande importance à l'élasticité de la peinture (effet de l'humidité sur le bois et craquelures).

Sur eiment, une couch'́ de protection au fluosilicate est à conseiller.

Si nous pouvons nous permettre un classement à la suite de ces essais, nous proposons :

- 10 Résines vinyliques;

- 20 Caoutehoue chloré;

- $3^{\circ}$ Phénolique cent pour cent.

\title{
UTILISATION DU LAIT DE BREBIS \\ EN ISRAEL (1)
}

\author{
par \\ le $\mathrm{D}^{r} \mathrm{KERN}$
}

Comme on l'a vu dans un premier article (2), on a réussi par un élevage méthodique à obtenir, en partant de la brebis primitive à grosse queue, une brebis laitière produisant presque autant de lait qu'une vache primitive.

Quand il est question des brebis laitières et de leurs produits, les lecteurs européens se souviennent tout d'abord du Roquefort français, du "Liptauer " des contrées carpathiques, du Caccio-

(1) Traduction C. Wolf, à qui Le Lait exprime à nouveau sa profonde gratitude.

(2) Le Lait, 1954, 335-336. 
Cavallo italien et du yoghourt bulgare. A l'origine, ces produits étaient préparés uniquement avec du lait de brebis ; mais, maintenant, le lait de vache est aussi bien utilisé que le lait de chèvre qui, dans beaucoup de pays, est considéré comme valant le lait de brebis, quoiqu'il en diffère complètement par sa composition. Dans la plupart des pays, le yoghourt n'est préparé qu'avec du lait de vache et se distingue entièrement du produit très acide, demi-solide, qui constitue le yoghourt original.

Alors qu'il existe de grands troupeaux de brebis laitières en Russie méridionale, en Turquie, en Arabie, et dans tous les pays de l'extrémité méridionale du bassin méditerranéen, les produits fromagers savoureux sont venus de la partie nord de ce bassin située dans un elimat tempéré. Je n'ai pas l'intention d'écrire une géographie du goût; mais je désire toutefois mentionner à ce sujet que, dans un climat tropical, une alimentation épicée et salée est préférée et il semble que dans de telles régions l'appréciation des nuances de goût plus différenciées soit perdue.

Le elimat sous-tropical de la Méditerranée n'a pas produit de variétés originales de fromage savoureux et l'imitation des variétés européennes de fromages en Israël a eu lieu comme une conséquence de l'immigration européenne : l'émigrant tient à son alimentation et à sa saveur propre, quoiqu'il soit plutôt douteux que la prochaine génération appréciera encore la chère de ses grandsparents ou s'adaptera mieux aux exigences du climat que la génération de la période de transition.

Ceci est également vrai en ce qui concerne le lait.

Presque tous les types européens de fromages ont été imités ici - toujours au prix de grands sacrifices financiers - et on est souvent arrivé à en obtenir d'excellentes qualités. L'industrie laitière primitive arabe fournissait au début de l'immigration juive tous les aliments nécessaires aux nouveaux venus. Après la construction des premières laiteries juives, les produits arabes furent imités et vendus par elles. Il faut toutefois noter ici que ces produits arabes n'ont de commun que le nom et les principes de fabrication avec les produits connus et consommés par les Fellahs et Bédouins indigènes; l'immigrant d'Europe les a entièrement modifiés au point de vue qualificatif par les méthodes modernes de production de ses laiteries. Il peut être toutefois intéressant de dresser la liste des produits originaux et de dire un mot de leur production, puisqu'on les prépare encore de la même façon dans tous les pays de la péninsule arabe, en Egypte et en Turquie.

D'après le Dr Hirsch (les moutons et les chèvres en Palestine Tel-Aviv, 1933), "le lait est un des produits principaux du mouton 
palestinien et les produits préparés avec ce lait, le beurre, le fromage, le "lebben" etc. sont les articles principaux de l'alimentation du pays. Le principal produit pour les Bédouins est le beurre, plus particulièrement préparé sous la forme de "semneh " (beurre fondu). Le "semneh" est préparé suivant deux méthodes distinctes, la première étant la préparation de beurre frais ou "sibde " de la façon suivante : le lait frais est versé dans une peau de chèvre contenant le reste de babeurre du jour précédent. Pendant la nuit, le lait devient acide et se coagule. Un peu avant le lever du soleil, a lieu le “barattage». La peau à moitié remplie est gonflée avec de l'air et secouée en avant et en arrière pendant une heure à une heure et demie... Ce beurre connu sous le nom de "sibde " est mou et malpropre. Il est sorti de la peau et, s'il y a en suffisamment, la fabrication du "semneh" suit immédiatement. S'il y en a peu, on le place dans un récipient avec du sel jusqu'à ce qu'une quantité suffisante ait été rassemblée. La fusion du "sibde" en vue de sa conservation s'opère comme suit : on allume un feu entre trois pierres et une grande cuve en cuivre est posée dessus, contenant, outre le "sibde» salé deux ou trois poignées de grains de safran et quelquefois des herbes aromatiques. Le tout est porté à l'ébullition jusqu'à ce que la graisse soit complètement clarifiée. Ce "semneh" préparé est placé dans une peau de chèvre où on le conserve (habituellement $20-25 \mathrm{~kg}$. dans une peau) pour être utilisé par la famille ou vendu. Le babeurre restant de la préparation du " sibde " est, soit bu frais, soit bouilli avec du riz. De plus grandes quantités sont transformées en une sorte de lait sec connu sous le nom de "Kishik». Pour la préparation du "Kishik", le babeurre est versé dans un sac en toile, on ajoute du sel, et le sac est suspendu pendant deux ou trois jours jusqu'à ce que l'eau soit évaporée. On moule des morceaux en forme de citron du produit demi-sec, morceaux qui sont ensuite desséchés au soleil jusqu'à ce qu'ils soient durs comme pierre et conservés pour être utilisés l'hiver et aussi pour la vente. Le " lebben " est préparé de la manière suivante : le lait frais est bouilli puis refroidi à une température telle qu'il soit tout juste possible d'y mettre le doigt. On y ajoute un "lebben " du jour précédent, on remue bien, puis on laisse au repos pendant cinq ou six heures. Les Fellahs produisent de grandes quantités de "lebben" aussi bien pour leur propre consommation que pour la vente au marché. Avec un "lebben " on prépare le "lebbenia" en versant du "lebben " dans un sac en toile que l'on pend afin que le liquide filtre. Le résidu est le "lebbenia" qui peut être mangé avec du pain ou moulé en morceaux et conservé dans l'huile d'olive. Le “lebbenia est quelquefois desséché au soleil et transformé en "Kishik " de la même manière que quand on le prépare avec du babeurre... 
La méthode arabe de préparation du fromage est très primitive. Au lait frais chaud, on ajoute un petit morceau de caillette de chèvre ou 'd'agneau. Quand le lait est caillé, la masse est placée dans un sac en toile afin que le sérum puisse s'égoutter. Ce qui reste est moulé en petits carrés et salé ou vendu frais au marehé, ou enfin conservé dans la saumure ".

De cette description, nous voyons que l'industrie laitière primitive s'efforce de transformer avec la vitesse la plus grande le produit facilement périssable en une forme plus durable. Comme il n'existe naturellement aucune possibilité de conserver et de réfrigérer, on utilise l'acidification (en utilisant l'acide lactique développé naturellement), le salage, la fusion et la dessication. Les souks arabes donnent toujours l'impression d'offrir un grand nombre de produits laitiers, car le même produit apparaît sur le marché sous diverses formes et avec de légères variations de goût : fromage salé en petits morceaux durs et plutôt secs (pas plus grands qu'un poing), grosses crêpes, babeurre cuit avec du riz et des épices, doux et âcre, etc. Une forme très intéressante et de bon goût du fromage salé se présente comme de petites boules marinées dans l'huile d'olive, pouvant se conserver pendant de nombreux mois, qui ne constituent un régal pour l'Européen qu'aussi longtemps que l'huile d'olive n'est pas devenue trop rance.

Le lait desséché, mentionné ci-dessus, et appelé iei "pierre de lait "n'a jamais été produit dans ce pays ; mais fut importé en grandes quantités pendant la deuxième guerre mondiale de divers pays voisins. En raison de sa forte teneur en sable, conséquence de sa dessication à l'air, ce produit n'a jamais convenu à la consommation humaine; mais on l'a souvent utilisé pour l'alimentation de la volaille après en avoir enlevé le sel par lavage. L'analyse du produit a donné une teneur en eau le plus souvent inférieure à $15 \%$,une teneur en sel entre $20 \%$ et $40 \%$; avec une forte teneur en sel, la composition était un peu celle d'un fromage salé sec ; mais quand la teneur en sel était faible, on trouvait des quantités considérables de lactose.

Le fromage "Brinsen » est un produit qui se rapproche le plus des anciens produits arabes. Il est préparé dans de vastes territoires de la Russie du sud, dans les Balkans, en Turquie, etc. Au début, il était produit dans les colonies juives d'après la recette ci-dessous : le lait de brebis était chauffé à environ $32^{\circ}$ et on ajoutait suffisamment de présure pour que le lait caille après une heure un quart, une heure et demie. Le caillé était immédiatement découpé et on en remplissait avec précaution et sans agitation les moules sur la plaque à égoutter. Les moules étaient garnis d'une toile et quand ils étaient remplis, on refermait la toile et un poids d'un demi kilo- 
gramme par litre était placé dessus ; après une demi-heure, le poids était doublé et le caillé restait dans cette presse primitive pendant huit heures, puis il était retourné et, après quatre autres heures, il était découpé et les morceaux placés en récipients (en bois ou en ciment) remplis d'une saumure de sel à $12 \%$. Après deux semaines, le fromage était emballé en boîtes métalliques contenant une saumure de sel à $14 \%$. Les colonies, c'est-à-dire les préparateurs du fromage, n'observaient ces prescriptions que très superficiellement ; ils apprirent vite à écrémer partiellement le lait et ne faisaient pas très attention au pourcentage de sel. Ainsi les laiteries qui s'occupaient du stockage et de la vente du fromage recevaient des produits qui, en apparence, en consistance et en composition, différaient considérablement les uns des autres. D'après des analyses effectuées pendant un certain nombre d'années, ce "Brinsen " avait la composition suivante :

$\begin{array}{lr}\text { Matière grasse entre: } & 13,5 \% \text { et } 34,5 \% \\ \text { Eau entre: } & 32,9 \% \text { et } 67,0 \% \\ \text { Extrait sec entre : } & 33,0 \% \text { ot } 67,1 \% \\ \text { Extrait sec dégraissé entre : } 32,9 \% \text { et } 62,2 \% \\ \text { Sel entre: } & 3,1 \% \text { et } 11,4 \%\end{array}$

Ce tableau donne un exemple classique d'un produit non normalisé. Les grandes différences résultent naturellement de la variation de la teneur en lait en matière grasse, ceci ne provenant pas autant de la variation naturelle de ce taux dans le lait que de l'écrémage du lait ou de l'addition de lait écrémé.

Afin de trouver une base à la normalisation du produit, de nombreux tests ont été effectués sur l'influence mutuelle de la teneur en matière grasse du lait de brebis pur et de la teneur en matière grasse de l'extrait sec du fromage. On a produit un "Brinsen" qui contenait à peu près exactement $50 \%$ d'eau et dont la teneur en sel (moyenne pour un morceau) était de $4,5 \%$. Le rapport suivant entre la teneur du lait de brebis en matière grasse et l'extrait sec du fromage est devenu net.

Le lait de brebis pur à $6,5 \%$ de matière grasse et plus devrait, d'après ce tableau, donner un fromage gaspillant la matière grasse, et il semble plus avantageux de produire le fromage avec du lait de brebis ne contenant pas plus de $4 \%$ de matière grasse, ce qui suppose une addition d'environ $50 \%$ de lait de brebis écrémé. Quand on prépare le fromage avec du lait de brebis pur, de grandes quantités de matière grasse partent dans le sérum; cette matière grasse peut être récupérée dans le sérum par les écrémeuses ; mais il est aussi devenu évident qu'une teneur trop élevée du lait en matière grasse n'améliore pas la qualité du fromage. Au contraire, il arrive 
Teneur $\%$ du leit en matière grasse

0,5
1,0
1,5
2,0
3,0
4,0
4,5
5,0
6,0
7,0
7,5
8,0
Teneur en matière grasse \% de l'extrait sec dufromage

6,5

12,4

16,7

23,0

30,0

36,5

42,5

43,0

47,8

52,3

54,5

56,8

quelquefois que la matière grasse suinte à la surface et que le fromage prenne une saveur acide. Dans la saumure des boîtes, nous avons trouvé jusqu'à $1,7 \%$ de matière grasse qui ne peut pas, cette fois, être récupérée. Actuellement, le "Brinsen " n'est plus produit que dans les laiteries et le fromage vendu est d'une qualité à peu près normale. La base de sa production repose sur une recette russe qui a subi quelques légères modifications.

Pour la production du "Brinsen ", tout lait de brebis ayant une acidité inférieure à $11,0 \mathrm{~S}$.-H. $(0,25 \%$ d'acide lactique) convient; l'acidité peut même être de 13,5 S.-H. $(0,31 \%$ d'acide lactique) à la fin de la période de lactation. Le lait doit être filtré immédiatement après la traite et ne doit pas contenir de colostrum ni avoir une saveur désagréable quelconque. Le lait est pasteurisé, car il a été reconnu que le lait pasteurisé donne un meilleur rendement et une meilleure qualité de fromage. Après refroidissement à $30-32^{\circ}$ (suivant la saison), on ajoute par 100 litres de lait $75 \mathrm{cc}$ d'une solution de chlorure de calcium à $40 \%$ et un litre d'un bon levain. On ajoute quelquefois aussi 30 grammes de salpêtre et suffisamment de présure (en poudre ou liquide) pour épaissir le'lait en 45 minutes. Le caillé doit être ferme et moelleux et le sérum d'un vert pur. On transfère à l'aide d'un puisoir plat le caillé aux tables d'égouttage en entassant couche sur couche jusqu'à une hauteur de 20 centimètres. Avant d'amener le caillé sous la presse, le fromager le retourne plusieurs fois. Au début du travail, le fromage est pressé très lentement ; mais, après 40 minutes, on augmente rapidement la pression jusqu'à ce qu'elle atteigne 15 kilogrammes par kilogramme de fromage. Le fromage est ensuite découpé en morceaux égaux qui sont mis dans une saumure qui a été préparée comme suit : 16 kilogrammes de sel bouilli dans 100 litres d'eau, puis filtration. Le bain salé est préparé frais chaque 
semaine en été et chaque quinzaine en hiver, Au fur et à mesure, le bain salé contient de plus en plus de sérum; mais la solution n'est pas changée aussi longtemps que la teneur en sel est suffisante. Le bain salé doit avoir une température de $18^{\circ}$. Après 48 heures, le fromage est sorti de la saumure et mis en boites métalliques propres contenant une solution salée à $12-14 \%$ de sel. Dans ces boîtes hermétiquement closes, le fromage peut être conservé frais jusqu'à 10 mois à une température moyenne de $15^{\circ}$. Un produit spécialement bon peut être obtenu avec du sérum cuit, et si avant d'ajouter le sel et de faire cuire le sérum on l'acidifie avec un levain. La teneur en sel du fromage lui-même ne doit pas être supérieure à $6 \%$. Ce genre de "Brinsen conservé dans la saumure " est commun ici alors que le "Brinsen sec " est préféré en Russie et dans d'autres pays. Ce dernier est préparé d'une façon similaire; mais la teneur en sel du bain salé doit être de $26 \%$ (sursaturé) et il doit toujours y avoir du sel non dissous au fond du récipient. Après 24 heures, le fromage est sorti de la saumure, salé avec du bon sel sec et empaqueté en fûts ou en caisses. Comme il perd encore de l'eau, il doit être réempaqueté s'il n'est pas immédiatement livré aux consommateurs.

Un fromage très similaire au "Brinsen sec " est produit ici sous le nom de "fromage de Safed ", dénommé d'après la ville de Safed d'où ce fromage est originaire. Il est aussi appelé "fromage de panier" parce qu'après le premier drainage, le caillé est mis en paniers d'osier tressé ; le fromage terminé est rond et reproduit nettement le dessin du panier d'osier. Quand on prépare ce fromage, le lait eșt mis en présure à $33-35^{\circ}$ de façon à rendre le caillé plus dur, ear, dans cette préparation, on n'utilise pas de presse. Immédiatement après l'écoulement du sérum, le fromage est retourné de panier à panier, étant chaque fois généreusement saupoudré de sel. Conservé en chambre froide, ce fromage, qui n'a pas de caractéristiques de fermentation, se conservera frais pendant plusieurs mois si on le sale de temps en temps.

Il n'y a pas grand chose à ajouter à propos du rendement du fromage salé. Si on utilise du lait de brebis pasteurisé non écrémé, le rendement s'élève à plus de 30 kilogrammes par 100 litres ; avec du lait partiellement écrémé, le rendement est approximativement de 25 kilogrammes.

Par suite de la grande insuffisance de lait qui s'est manifestée au cours des dernières années, on a ajouté de plus en plus de poudre de lait, et quand on a constaté que les produits obtenus étaient entièrement satisfaisants; la proportion de lait frais est devenue de plus en plus minime et actuellement le "Brinsen " et le "Safed" sont obtenus avee un lait contenant $90 \%$ d'une solution de poudre 
de lait, 10\% de lait frais et de la graisse végétale. La graisse végétale est homogénéisée avec une partie de la solution de poudre de lait et mélangée ainsi au fromage. De cette façon, il a été possible de produire du "Brinsen" pendant tous les mois de l'année et cela $a_{a}$ permis d'utiliser au mieux la poudre de lait écrémé importée.

Une des laiteries locales produit, en quantités variables, un "fromage bleu " (genre Roquefort). Ce fromage est d'une qualité relativement supérieure et, pendant la deuxième guerre mondiale, fut exporté en Egypte, où il fut servi sur la table du Roi. Il n'est pas nécessaire, dans une revue française, de s'étendre sur la production d'un fromage genre Roquefort. On doit seulement mentionner qu'un produit très satisfaisant a été obtenu en imitant les conditions climatiques naturelles dans de grandes chambres refroidies avec une certaine humidité. Les frais de production sont plutôt élevés en raison du capital important nécessairement investi et de la longue période de maturation, de sorte que ce fromage est assez cher et sa fabrication plutôt restreinte. Une analyse typique de ce fromage est la suivante :

$29,3 \%$ de matière grasse ; $46,5 \%$ de protéine ; $37,5 \%$ d'eau ; $47,0 \%$ de matière grasse dans l'extrait sec.

Dans tous les pays balkaniques, aussi bien en Turquie qu'en Italie, on produit du Caccio-Cavallo (Kashkaval) et grâce à sa longue conservation, même à une température extérieure élevée, c'est le fromage favori de ces pays. C'est certainement le fromage à teneur en eau la moins élevée, teneur pouvant descendre jusqu'à $20 \%$. Le "Kashkaval " arrive au marché en pains plats minces de 3 à 6 kilogrammes. Dans la production de cette sorte de fromage, après le caillage habituel (30 à 40 minutes), le caillé est chauffé à $80-85^{\circ}$ dans le sérum, ou bien on évacue le sérum et on chauffe le caillé dans l'eau jusqu'à la température ci-dessus indiquée. On obtient avec le caillé une substance pâteuse, caoutchouteuse. Après que la masse a été mise en moules, le fromage est de nouveau pressé pour réduire au minimum la teneur en eau. Le fromage n'est salé que par l'extérieur et, après quatre ou einq mois, il est prêt pour la vente. Quand on prépare cette sorte de fromage, il faut ajouter généreusement de la matière grasse, sinon le fromage devient cassant et n'a pas le bon goût typique. L'addition de solution de poudre de lait n'est pas possible avec cette sorte de fromage, sinon le caillé serait cassant quand on le chaufferait et ne pourrait pas être traité. Le "kashkaval " est préparé en quantités relativement importantes, surtout par les petites laiteries et les immigrants des Balkans. 
La production des autres sortes de fromages provenant du lait de brebis n'a jamais dépassé le stade préliminaire primitif et ee chapitre de la production du fromage peut être arrêté ici. Des quantités appréciables de lait de brebis sont, toutefois, ajoutées au lait de vache dans la fabrication de diverses variétés de fromages, sạns nuire en quoi que ce soit à la qualité. On peut ajouter à un " fromage hollandais " préparé ici en grandes quantités, $25 \%$ de lait de brebis, au Romadur et au Limburger plus de $40 \%$, etc.

Je voudrais ajouter ici quelques détails sur la production et la consommation du fromage en Israël. La production de l'an dernier a été d'après les statistiques de :

-4.730 tonnes de fromage blanc mou, préparé presque exclusivement avec de la poudre de lait écrémé. Teneur en eau autorisée jusqu'à $78 \%$.

- 1.500 tonnes de fromage blanc à la crème, préparé avec du lait écrémé en poudre et de la graisse végétale.

- Teneur en eau autorisée jusqu'à $75 \%$; teneur minimum en matière grasse : $9,0 \%$.

- 910 tonnes de fromage fondu préparé avec du fromage importé et de la poudre de lait.

-1.528 tonnes des autres sortes de fromages "Brinsen"; "Safed ", un fromage genre Edam et un genre Roquefort, Romadur et Limburger, etc.

Comme on le voit par ces chiffres, la consommation de fromage est assez élevée ( $7 \mathrm{~kg}$. 0 par tête et par an) et la moitió de cette quantité est constituée par du fromage blanc frais. Ce fromage diffère du fromage de cottage américain en ce qu'il possède une saveur aigrelette et une consistance quelque peu pâteuse, mais pas trop onctueuse. Il est préparé avec du lait écrémé additionné d'un bon levain et d'une petite quantité de présure et de chlorure de calcium. Il n'est ni réchauffé ni lavé. Pendant les mois d'été, il se développe quelquefois une sur-acidité (acide acétique). Ce fromage frais peut aussi être préparé avec du lait de brebis sans aucune différence de saveur et de consistance; mais il est de consistance plus fine et plus onctueuse que le fromage de lait de vache. La pasteurisation à haute température est très avantageuse pour la qualité, car, dans ce cas, une grande partie de l'albumine est absorbée dans le fromage, améliorant la qualité et augmentant considérablement la quantité. J'ai déjà indiqué dans la partie théorique de cette étude la question du rendement et ai aussi expliqué la nécessité d'utiliser le sérum du lait de brebis par la production de fromage albumineux. On ne procède à cette fabrication du fro- 
mage que dans quelques laiteries en chauffant et en acidifiant le sérum.

L'industrie laitière juive ne connaît que quelques variétés de fromage de brebis et ne produit pas de beurre de lait de brebis, ni de lait de brebis de consommation, ni de produits de laits acides spécifiques du lait de brebis. On n'a produit du beurre de lait de brebis que pour la recherche afin de démontrer des insuffisances dans la qualité du beurre. Comme les fabricants de fromage des colonies juives écrèment quelquefois le lait de brebis utilisé pour la fabrication du "Brinsen ", des quantités appréciables de crème de lait de brebis arrivaient aux laiteries qui le transformaient en beurre avec de la crème de lait de vache. Comme, toutefois, la crème absorbe toute odeur particulière, d'une façon même plus intense que le lait, et comme le lait de brebis est en général moins propre que le lait de vache, une bonne partie des défauts du beurre a pu être attribuée au mélange aveo du lait de brebis. Toutefois, dès que les laiteries, au lieu des colonies, commencèrent à écrémer le lait, les plaintes au sujet de la qualité du beurre devinrent moins nombreuses. Pendant de nombreuses années, les laiteries refusèrent d'accepter les laits de brebis en raison de leur plus forte pollution et de leur malpropreté. Du reste, les écrémeuses et les pasteurisateurs à plaques étaient souvent engorgés en raison de la viscosité élevée du lait de brebis. Les surplus temporaires de lait de brebis sont maintenant fortement dilués avec de l'eau, centrifugés, pasteurisés et transformés en crème, beurre ou en fromage de lait écrémé. On recerait quelquefois encore des plaintes à propos de la saveur du beurre; mais aucune à propos du fromage. C'est pourquoi pendant toute une période de lactation, on a produit du beurre de lait de brebis et on l'a analysé comme indiqué dans la partie théorique de cet article. Au début de la période de lactation (décembrejanvier), le beurre de lait de brebis est d'une qualité plus onctueuse et plus pâteuse que le beurre ordinaire, quoique n'en différant pas comme saveur ; de la fin d'avril à juin, nous avons toujours trouvé que le beurre avait de plus grandes tendances à devenir rance. La consistance du beurre pendant cette période est toutefois absolument la même que celle du beurre de vache. Au cours des récentes annés, quand le lait et les produits laitiers étaient très rares en Israël, de la crème de lait de brebis était ajoutée à la crème du lait de vache et on n'a jamais reçu de plainte relative à la qualité. La crème était entièrement lavée, on employait un levain de première qualité, la température de fermentation était plus basse que celle utilisée d'habitude pour permettre une fermentation très lente et le beurre était baratté avec une crème à teneur en matière grasseinférieure à $30 \%$. 
Certaines quantités de lait de brebis sont naturellement mélangées au lait de consommation. Il faut mentionner ici qu'il n'existe aucun troupeau arabe de brebis qui ne soit mélangé de chèvres. Les colonies juives avaient aussi l'habitude de mélanger les deux sortes de laits. Ce mélange avec du lait de chèvre est un fait des plus regrettables au point de vue hygiénique. Les laiteries ont fait tous leurs efforts pour empêcher cette pratique; mais n'y réussirent que dans les grandes colonies. Si du lait de brebis pur doit être utilisé comme lait de consommation, on peut le diluer avee environ $50 \%$ d'eau ; on obtient ainsi un produit très similaire en ce qui concerne les teneurs en matière grasse et en protéine. Quand un tel lait a bouilli, la saveur et l'arome désagréables (qui sont souvent caractéristiques du lait de brebis en raison de la traite et du traitement ultérieur effectués dans des conditions peu hygiéniques) disparaissent. Un tel lait ne doit pas être donné aux enfants en raison de sa faible teneur en substances minérales et en vitamines résultant du fort mélange d'eau.

En ce qui concerne l'odeur et la saveur, l'auteur veut ajouter d'après sa propre expérience que le lait de brebis produit dans des conditions hygiéniques est une boisson savoureuse et n'est pas inférieur au lait de vache. La saveur particulière est partiellement due à la plus forte viscosité. Quand cette viscosité est réduite par la dilution, le lait de brebis peut à peine être distingué du lait de vache. En Israël, comme dans beaucoup d'autres pays, la traite de la brebis est effectuée par l'arrière. C'est en raison de cette particularité et du fait que la période de traite de la brebis coïncide avec la saison pluvieuse et boueuse, que le lait de brebis sera toujours moins propre (macroscopiquement et microscopiquement) que le lait de vache. Malgré de nombreux avis et des eampagnes explicatives contre la traite par l'arrière, cette pratique n'a pas encore été modifiée.

\section{Produits lactés acides}

Ce paragraphe ne traite pas seulement des produits du lait de brebis, mais aussi de ceux du lait de vache préparés dans ce pays.

Yoghourt. - Ce produit très important du lait de brebis dans les Balkans et en Turquie n'a pas eu de succès en Israël. Dans la plupart des pays de haute culture, le yoghourt est préparé en quantités énormes avec le lait de vache, et l'immigrant juif l'a appris dans son pays d'origine. La raison pour laquelle le yoghourt n'a pas pu pénétrer sur notre marché réside probablement dans le fait qu'au temps de la grande immigration (vers les années 1930), les magasins de nos villes n'étaient pas encore munis de réfrigérateurs néces- 
saires, ce qui avait pour résultat que les consommateurs recevaient trop souvent un yoghourt déjà trop acidifié. En raison de la croissance rapide des bactéries acidophiles, le Yoghourt devient plus rapidement acide que le "lebben ", produit laitier qui sera décrit plus loin. Les essais faits pour vendre un yoghourt convenablement concentré d'après la méthode turque, ne réussirent pas non plus pour une autre raison. La saison pour les produits lactés acides débute dans ce pays vers le milieu de mai et à cette époque les rendements en lait de brebis sont déjà, pour la plupart, en diminution, de sorte que la production du yoghourt avec du lait de brebis n'est pas possible. Le yoghourt préparé avec du lait de vache tend encore plus, dans notre climat chaud, à s'acidifier que le lait de brebis. On a ainsi souvent essayé d'introduire sur une grande échelle le yoghourt sur notre marché. Seuls les immigrants des Balkans ont réussi à en vendre au cours des dernières années ; mais cette vente, jusqu'à maintenant, est restée plutôt faible. Les échantillons analysés contiennent jusqu'à $23 \%$ d'extrait sec et $4 \%$ de matière grasse. Le lait original consistait soit totalement, ou pour la plus grande partie en une solution de poudre de lait. Ce genre de yoghourt a, en raison de sa forte teneur en lactose, une saveur un peu douceâtre et constitue un produit demi-solide ressemblant à du fromage et qui est extrêmement acide.

Lebben. - Jusqu'à il y a dix ans, le "lebben» était le plus populaire des produits lactés acides vendus dans ce pays. Le "lebben " arabe, préparé presque uniquement avec des laits de brebis et de chèvre, a été imité par les immigrants juifs vers 1920 ; mais était préparé avec du lait de vache et produit dans les laiteries. Il était préparé d'après la méthode suivante : le lait était fortement chauffé au début dans un pasteurisateur chambreur pendant 10 à 20 minutes à $85-90^{\circ}$; mais par la suite, la période de chauffage a été réduite et les laiteries ont réussi à préparer un produit parfait avec du lait chauffé pendant un temps assez court. Le lait était ensuite refroidi à $23^{\circ}$ en été et à environ $28^{\circ}$ en hiver et ensemencé avec un bon levain (Bac. cremoris et lactis, organismes mélangés utilisés pour l'acidification de la crème). Le lait était ensuite mis en bouteilles, analogues à celles du yoghourt, qui étaient transférées dans des salles convenablement chauffées. Après 5 à 7 heures, le lait commençait à se raffermir d'une façon homogène dans les récipients et le "lebben " ainsi terminé était transféré en chambre froide. Son acidité ne devait pas être inférieure à $36^{\circ}, 0$ S.-H. $\left(0,82 \%\right.$ d'acide lactique) ni supérieure à $40^{\circ}, 0$ S.-H. $(0,92 \%$ d'acide lactique). Le consommateur désirait surtout que ce produit soit constitué par une masse ferme et parfaitement homogène d'une saveur légèrement acide sans séparation de sérum et sans arome 
exagéré. On trouve en vente diverses variétés de "lebben ". A un moment il y en avait quatre sortes différentes dont une première qualité avec $2,8 \%$ de matière grasse ; une autre avec $1,7 \%$ et une préparée avec du lait écrémé. Il y avait en outre du lait acidifié mis en bouteilles bien fermées et marquées "Kefir ". Le "lebben" préparé avec du lait écrémé ne restait jamais longtemps en vente ; le lait n'étant pas concentré avait une grande tendanee à libérer du rérum et sa saveur était plutôt fade. L'addition de seulement $1 \%$ de matière grasse dans le lait est suffisante pour donner un produit tout à fait savoureux. Aujourd'hui, le "lebben" est composé de $1,2 \%$ de graisse végétale et de $10 \%$ de poudre de lait écrémé. L'utilisation de la poudre de lait permet d'atteindre facilement la consistance du produit arabe, qui est obtenue par une longue cuisson et l'addition de lait de brebis ; les laiteries juives utilisent aussi occasionnellement du lait de brebis dans la production du "lebben " sans intention de préparation d'un produit spécial. Depuis que le "lebbenia " a été mis en vente, la vente du "lebben " a beaucoup diminué, n'atteignant qu'à peine 100.000 litres cette année.

Crème acide, - Un autre produit typique de ce pays qui a disparu du marché (mais qui, à mon avis, contrairement au "lebben ", y reviendra) est la crème acide, lait acide de très bonne qualité préparé comme le "lebben ", mais contenant $22,0 \%$ de matière grasse. Ce produit était vendu en gobelets en carton de 50 et 100 grammes et était d'une consistance fluide, mais épaisse et homogène, avec une saveur très aromatique. Le levain utilisé pour ce produit était le "levain pour beurre" déjà mentionné. En 1942, la Commission mandataire du Gouvernement britannique interdisait la fabrication de ce produit, quoiqu'il y eut encore à ce moment des quantités suffisamment disponibles de matière grasse de lait dans le pays.

Lebbenia. - Au cours de la recherche d'un succédané pour cette crème acide, je suggérai la fabrication du "lebbenia ». Le " lebbenia » arabe est une combinaison de lait acide (lebben) et de fromage blanc mou, et est obtenu en enlevant une partie du sérum contenu dans le "lebben ". Considérant que la population devait au moins recevoir, au lieu de la concentration de matière grasse (crème) interdite, une concentration de protéine, je préparai un produit lacté acide qui, par l'addition de caillé, avait une forte teneur en protéine. En homogénéisant les deux composants : lait acide et caillé, on obtint un produit savoureux et très intéressant. L'homogénéisation devint un des facteurs principaux de l'obtention d'une consistance crémeuse et fut suffisante pour coaguler et homogénéiser du lait à $3,6 \%$ ou plus de matière grasse et obtenir le produit 
désiré. Ceci signifie que nous pouvions abandonner l'addition du caillé au lait; plus le lait était concentré, meilleur était le "lebbenia ". Il est surtout facile de l'obtenir du lait de brebis, puisque le produit de départ a une forte teneur en protéine. Le mode actuel de préparation du "lebbenia" exige $15 \%$ de matières solides consistant en $11,0 \%$ de poudre de lait écrémé et $4,0 \%$ de graisse végétale. En utilisant de la poudre de lait, nous nous rapprochons de nouveau de l'idée de début de donner plus de protéine à la population. Le "lebbenia " d'avant guerre (jusqu'à 1948) pouvait être défini comme un "babeurre concentré et fermenté" d'une teneur en matière grasse de $4 \%$ et d'une consistance crémeuse. En moins de dix ans, le "lebbenia " a conquis le marché local et l'an dernier, il en a été produit 20 millions de litres... J'ai mentionné ici tous les produits lactés d'Israël quoiqu'ils ne soient préparés qu'avec un très faible pourcentage de lait de brebis.

Les experts ainsi que les hommes de science seront frappés par le fait que tous les produits lactés peuvent être préparés avec de la poudre de lait. Au cours de ces dernières années, Israël a été comme un grand laboratoire où on a procédé à des essais pour trouver comment alimenter une population dense avec de la poudre de lait à des moments de déficit presque complet de ravitaillement en lait naturel.

Avec le ralentissement de l'arrivée de nouveaux immigrants et l'augmentation de la production laitière d'Israël, l'utilisation de la poudre de lait diminuera de nouveau et le problème de la production laitière locale et de l'utilisation de cette production deviendra de nouveau une question d'une grande importance.

A ces problèmes appartient aussi la question du paiement du lait, y compris le lait de brebis. Depuis plus de vingt ans, tout le lait de vache provenant des colonies juives a été payé d'après sa teneur en matière grasse, une teneur de $3,5 \%$ étant considérée comme celle d'un "lait normal " et des prix plus bas ou plus élevés étant payés suivant que le taux de matière grasse était inférieur ou supérieur à ces $3,5 \%$. Dans les plus grandes laiteries, des échantillons sont prélevés tous les jours sur chaque réception.

Quelquefois, le paiement avait lieu d'après la qualité (acidité, propreté, qualité bactériologique); mais cette méthode n'est plus utilisée maintenant. Le paiement à la qualité sera à examiner de nouveau à la lueur d'une production suffisante ou excédentaire. La rareté a toujours une mauvaise influence sur la qualité...

Le lait de brebis a été pendant de longues années traité en "orphelin", en ce qui concerne le paiement : quelquefois, il était payé sans considération de la teneur en matière grasse sur la base de $3,5 \%$ du lait de vache, quelquefois même à des prix plus réduits. 
Au cours de ces dernières années, et en raison de ce que des quantités considérables de crème de lait de brebis ont été utilisées pour la production du beurre, on le paie d'après sa véritable teneur en matière grasse. Il en résulte que les éleveurs de moutons reçoivent aujourd'hui pour leurs produits un prix supérieur de près de $50 \%$ aux fournisseurs de lait de vache. Les éleveurs de moutons s'efforcent aussi de recevoir des prix encore plus élevés en raison de la teneur en caséine du lait de brebis. Toutefois, les laiteries maintiennent, avec raison, qu'il existe des difficultés dans le traitement du lait de brebis et que si le lait de brebis était payé à la qualité (propreté, qualité bactériologique, ete.) la réduction serait bien plus grande que dans le cas du lait de vache. Ce problème sera très probablement réglé dès que des laiteries spéciales pour lait de brebis auront été construites ou qu'une séparation complète du lait de brebis et du lait de vache aura été effectuée dans les laiteries actuelles. Alors, un juste paiement pourra être effectué d'après les produits manufacturés; les prix pour le lait de brebis seront complètement indépendants de ceux du lait de vache et l'évaluation en sera faite exactement aussi bien pour le fournisseur que pour les fabricants, e'est-à-dire les laiteries.

Nous devons nous attendre à une forte augmentation de la production du lait de brebis dans les années à venir et un prix convenable est à la base de ce progrès, comme à celle de tout autre secteur économique.

\title{
LE LAIT DANS LE MONDE
}

\author{
par \\ G. GÉNIN \\ Ingéniẹur E. P. C.
}

\section{ANGLETERRE}

\section{La fabrication des fibres de caséine}

La fibre de caséine, qui avait été fabriquée avant la guerre par la Courtaulds Ltd sur une échelle réduite est maintenant produite d'une façon beaucoup plus importante dans une nouvelle usine construite par cette firme à Coventry et est vendue à l'industrie textile sous le nom de fibre Fibrolan.

La caséine lactique utilisée comme matière première est beaucoup plus chère qu'elle l'était avant guerre, mais les améliorations apportées au produit ont permis néanmoins de la fournir à l'industrie ầ un prix qui est le plus bas de toutes les fibres protéines, qu'il s'agisse de fibres naturelles ou de fibres artificielles.

La caséine, qui doit répondre à des normes rigoureuses en ce qui 